\section{Not enough evidence to include ESR1 amplification}

\author{
Christoforos Thomas and Jan-Åke Gustafsson
}

In our recent Review (The different roles of ER subtypes in cancer biology and therapy. Nature Rev. Cancer 11, 597-608 (2011)) ${ }^{1}$, we address the mechanisms that regulate the cellular levels of oestrogen receptors (ERs). We thank Yu et al. for their correspondence on our article ${ }^{2}$, in which we focus on the role of the transcriptional regulation of ERs, including the hypermethylation of ER promoters, the post-transcriptional regulation by microRNAs and proteasomemediated degradation of ERs. Alteration of ER levels by these mechanisms has been reported to be a common event in a number of cancers (such as in breast, prostate, colon and gynaecological cancers). In addition, the contribution of these mechanisms to the regulation of the ER levels in cancer has been validated in different systems (specimens from human subjects, and animal and cell-based studies) by using different methods ${ }^{3-6}$.

In their correspondence, Yu et al. indicate that the mechanism of ESR 1 amplification, which is not mentioned in our Review, deserves to be discussed when illustrating the mechanisms of ER $\alpha$ upregulation. To support this, they discuss the results from several studies, including that of Holst et al. ${ }^{7}$, which reported that amplification of ESR1, as detected by fluorescence in situ hybridization (FISH), occurs in a substantial proportion of breast cancers (20.6\%). Holst et al. ${ }^{7}$ also found that ESR1 amplification predicts a better response to tamoxifen therapy in a subset of individuals with breast cancer ${ }^{7}$. However, several groups have challenged these results, and, by using a variety of methods including comparative genomic hybridization (CGH), FISH and quantitative PCR, have shown a low frequency of ESR 1 amplification in breast cancers (two studies reported ESR1 amplification in 1\%, and less than $1 \%$, of breast cancers $)^{8-11}$. Interestingly, in one of these studies, ESR1 amplification did not show any significant correlation with ERa expression ${ }^{10}$. More recent studies have produced contradictory data with regard to the frequency of ESR 1 amplification (different ESR1 amplification frequencies have been detected with different techniques) and have questioned its clinical significance for breast and other types of cancer ${ }^{12}$. Consistent with the data from the majority of the previous studies, Moelans et al. ${ }^{13}$ have recently shown that ESR1 amplification is a rare event in breast cancer. In contrast to the findings by Holst et al. ${ }^{7}$, Nielsen et al. ${ }^{14}$ suggested that ESR1 amplification is associated with increased tamoxifen resistance in ER $\alpha$-positive breast cancers. Together, these results, which have been derived from several institutions, have not validated previous findings of high-frequency ESR 1 amplification in cancer ${ }^{7}$ and conclude that ESR1 amplification in breast cancer is an uncommon event of unknown clinical importance.

Taking these data into consideration in our Review, we avoided including ESR1 amplification in the list tabulating important and wellvalidated mechanisms that are responsible for changes in ER levels and activities in cancer cells and tissues.
Christoforos Thomas and Jan-Åke Gustafsson are at the Center for Nuclear Receptors and Cell Signalling, Department of Biology and Biochemistry, University of Houston, Houston 77204, Texas, USA.

Jan-Åke Gustafsson is also at the Department of Biosciences and Nutrition, Novum, Karolinska Institute, Huddinge S-14183, Sweden.

Correspondence to J.-A.G. e-mail: igustafs@Central.UH.EDU

doi:10.1038/nrc3093-c2

Thomas, C. \& Gustafsson, J.-Å. The different roles of ER subtypes in cancer biology and therapy. Nature Rev. Cancer 11, 597-608 (2011).

2. Yu. et al. ESR 1 gene amplification: another mechanism regulating the cellular levels of ERa. Nature Rev. Cancer (doi: 10.1038/nrc3093-c1).

3. Pan, X. et al. Elevated expression of CUEDC2 protein confers endocrine resistance in breast cancer. Nature Med. 17, 708-714 (2011).

4. Giamas, G. et al. Kinome screening for regulators of the estrogen receptor identifies LMTK3 as a new therapeutic target in breast cancer. Nature Med. 17 715-719 (2011).

5. Al-Nakhle, H. et al. Estrogen receptor $\beta 1$ expression is regulated by miR-92 in breast cancer. Cancer Res. 70 4778-4784 (2010)

6. Zhu, X. et al. Dynamic regulation of estrogen receptorbeta expression by DNA methylation during prostate cancer development and metastasis. Am. J. Pathol. 164, 2003-2012 (2004).

7. Holst, F. et al. Estrogen receptor alpha (ESR1) gene amplification is frequent in breast cancer. Nature Genet. 39, 655-660 (2007).

8. Brown, L. A. et al. ESR1 gene amplification in breast cancer: a common phenomenon? Nature Genet. 40, 806-812 (2008)

9. Horlings, H. M. et al. ESR1 gene amplification in breast cancer: a common phenomenon? Nature Genet. 40, 807-812 (2008).

10. Reis-Filho, J. S. et al. ESR1 gene amplification in breast cancer: a common phenomenon? Nature Genet. 40, 809-812 (2008).

11. Vincent-Salomon, A., Raynal, V., Lucchesi, C., Gruel, N. $£$ Delattre, O. ESR 1 gene amplification in breast cancer: a common phenomenon? Nature Genet. $\mathbf{4 0}$ 809-812 (2008).

12. Tan, D. S., Lambros, M. B., Marchio, C. \& Reis-Filho, J. S. ESR1 amplification in endometrial carcinomas: hope or hyperbole? J. Path. 216, 271-274 (2008).

13. Moelans, C. B. et al. ESR 1 amplification is rare in breast cancer and is associated with high grade and high proliferation: a multiplex ligation-dependent probe amplification study. Cell Oncol. (Dordr) (doi:10.1007/s13402-011-0045-5).

14. Nielsen, K. V. et al. Amplification of ESR1 may predict resistance to adjuvant tamoxifen in postmenopausal patients with hormone recepto positive breast cancer. Breast Cancer Res. Treat 127 345-355 (2011)

Competing interests statement

J.-A.G. is a consultant with Karo Bio AB and BioNovo. C.T declares no competing financial interests. 